\title{
CHAOS AND QUANTUM MECHANICS
}

\author{
Salman Habib \\ MS B285, Theoretical Division, The University of California, Los Alamos National Laboratory, \\ Los Alamos, New Mexico 87545 \\ habib@lanl.gov \\ Tanmoy Bhattacharya, ${ }^{1}$ Benjamin Greenbaum, ${ }^{2}$ Kurt Jacobs,,${ }^{1,3}$ \\ Kosuke Shizume, ${ }^{4}$ and Bala Sundaram, ${ }^{5}$ \\ ${ }^{1}$ Los Alamos National Laboratory, ${ }^{2}$ Columbia University, ${ }^{3}$ Griffith University, ${ }^{4}$ Tsukuba Uni- \\ versity, ${ }^{5}$ City University of New York
}

\begin{abstract}
The relationship between chaos and quantum mechanics has been somewhat uneasy - even stormy, in the minds of some people. However, much of the confusion may stem from inappropriate comparisons using formal analyses. In contrast, our starting point here is that a complete dynamical description requires a full understanding of the evolution of measured systems, necessary to explain actual experimental results. This is of course true, both classically and quantum mechanically. Because the evolution of the physical state is now conditioned on measurement results, the dynamics of such systems is intrinsically nonlinear even at the level of distribution functions. Due to this feature, the physically more complete treatment reveals the existence of dynamical regimes - such as chaos - that have no direct counterpart in the linear (unobserved) case. Moreover, this treatment allows for understanding how an effective classical behavior can result from the dynamics of an observed quantum system, both at the level of trajectories as well as distribution functions. Finally, we have the striking prediction that time-series from measured quantum systems can be chaotic far from the classical regime, with Lyapunov exponents differing from their classical values. These predictions can be tested in next-generation experiments.
\end{abstract}

\section{Prologue}

I met Henry Kandrup as a graduate student at Maryland in 1985, having recently decided to switch from experiment to theory. My first interaction with postdocs - at the time an intimidatingly higher form of life - occurred when Henry suggested that he and another postdoc, Ping Yip, and I take up the 
question of Landau damping and stability of star clusters. While I was happy to work with Henry and Ping, most of the time I was struggling to understand cryptic conversations laced with mathematical jargon - "functions of compact support," "consider the following inner product," and so on. Since I wasn't following too much of this, I decided it was better to go away and catch up by reading every paper that was even vaguely related to the topic. This turned out to be much easier than expected, and one night I came up with a simple way of combining Henry's previous work on stability with conventional Landau damping theory from plasma physics. Coming in to the department late in the morning I showed the first set of notes to Henry. He looked at them, did not say much - which was unusual - and went back home. Next day, as I entered my office, I was stunned to find, slipped under the door, a complete preprint of a paper, all equations written in by hand in Henry's beautiful copperplate. He had gone home, generalized my notes to the problem at hand, worked through the entire thing, come back late at night, and typed the preprint on an electric typewriter (this was just before the advent of word processing), finishing as the sun came up. After this incident, I was really afraid of postdocs!

My early interactions with Henry were very wide-ranging; we discussed all sorts of topics, from classical statistical mechanics to quantum gravity, and on all of them he was very well-informed and entertainingly opinionated. The years went by quickly, Henry moved on to other places and so did I. Although we argued and collaborated now and then as of old, in my memory the early years have a certain luminescence. My favorite remembrance of Henry is that after he had demolished somebody's hapless piece of research in one of our discussions, he would look up, smile in a disarming way, and say, "True?" It usually was.

One of the topics Henry and I discussed at considerable length and depth was the nature of chaos in multi-particle systems and its role in controlling aspects of the dynamical behavior of statistical averages. While we did not always agree, these discussions certainly attuned my thinking about the problem. In this contribution, I present a discussion of how to think about chaos in a physical way, from the point of view of realistic experiments. The basis of the arguments applies to both classical and quantum systems and serves to bring together these two great dynamical traditions that are seemingly at such odds with each other. The work reported here is the result of several collaborations between subsets of the authors. While I do not know what Henry's opinions would have been on this subject, however, I am sure he would not have been quiet! 


\section{Introduction}

In classical theory - unlike in quantum mechanics - the status of dynamical chaos is apparently clear: chaos exists observationally and is well-described theoretically by Newton's equations. (Nevertheless, even here, a deeper look at the physical meaning of chaos is certainly helpful; we return to this presently.) It is in the context of quantum theory, however, that the notion of chaos appears so puzzling and mysterious. Because of the Kosloff-Rice theorem [1] and related results [2], it is clear that quantum evolution of the wave function or the density matrix is integrable; hence, chaos cannot exist in quantum mechanics in the canonical sense. This is the basic stumbling block to defining a quantum notion of nonintegrability.

One may argue that real quantum systems are always coupled to an environment and hence their evolution - "for all practical purposes," (FAPP), in Bell's famous phrase [3] - should be described by unitarity-breaking master equations rather than the unitary evolution assumed by the Kosloff-Rice theorem. Perhaps this way out, although not fundamentally satisfying to the purist, is enough by itself, but it is easy to see what is wrong with the argument. Fundamentally, any fully quantum dynamical description must arise from a Hamiltonian describing the system, its environment, and their coupling. The master equation represents the evolution of the reduced density matrix for the system which arises from tracing over the environment variables in the full (system plus environment) density matrix. Since the full evolution must satisfy Kosloff-Rice, the evolution of the reduced density matrix cannot be nonintegrable.

Thus, the fundamental problem we are faced with is this: we are familiar with chaos in the real world, but our fundamental theory of dynamics - which passes every experimental test beautifully - seemingly does not have a natural place within it to tolerate even the existence of the concept. This should not come as a surprise; after all, the trajectories of classical mechanics are apparently "real" and effortless to contemplate, but they too, have no natural place in quantum mechanics. Now it is true that quantum mechanics is an intrinsically probabilistic theory, but that, in itself, is not the real issue. Classical theory can be easily cast as fundamentally probabilistic as well, via the classical Liouville equation describing the evolution of a classical probability in phase space. (For an attempt at an even closer analogy, see Ref. [4] and the discussion in Ref. [5].) As discussed further below, the key point is rather that, unlike special relativity, where $v / c \rightarrow 0$ smoothly transitions between Einstein and Newton, the limit $\hbar \rightarrow 0$ is singular. The symmetries underlying quantum and classical dynamics - unitarity and symplecticity, respectively - are fundamentally incompatible with the opposing theory's notion of a physical state: 
quantum-mechanically, a positive semidefinite density matrix; classically, a positive phase-space distribution function.

In the rest of this article, we will expose the singular nature of the $\hbar \rightarrow 0$ limit and discuss a physical point of view - applicable to both classical and quantum systems - which will enable us to explain how trajectories and chaos appear in real experiments.

At this point, it should be clear that the questions taken up in this contribution are not those usually considered under the research area called "quantum chaos." There, one is primarily interested in the quantum behavior of a system with a classically chaotic Hamiltonian, what might happen to the validity of certain approximations (e.g., semiclassical approaches to calculating the quantum propagator) and whether classical trajectories and phase space structures can provide some insight into the nature of quantum wavefunctions. But one does not actually study quantum chaos.

We distinguish between isolated evolution, where the system state evolves without any coupling to the external world, unconditioned open evolution, where the system evolves coupled to an external environment but where no information regarding the system is extracted from the environment, and conditioned open evolution where such information is extracted. In the third case, the evolution of the physical state is driven by the system evolution, the coupling to the external world, and by the fact that observational information regarding the state has been obtained. This last aspect - system evolution conditioned on the measurement results via Bayesian inference - leads to an intrinsically nonlinear evolution for the system state, and distinguishes it from unconditioned evolution. While the concept of conditioned evolution of the system state is familiar to engineers and mathematicians, especially systems engineers and control theorists [6], it is not yet completely familiar territory to the majority of physicists. Nevertheless, driven by the impressive progress in the experimental state-of-the-art in quantum and atomic optics and in nanoscience [7], these notions are now being employed as everyday tools at least in some fields.

The conditioned evolution provides, in principle, the most realistic possible description of an experiment. To the extent that quantum and classical mechanics are eventually just methodological tools to explain and predict the results of experiments, this is the proper context in which to compare them and discuss the nature of predictions for real experiments. The explicit incorporation of information gained via measurement also provides a structure to address the quantum-classical transition more generally, and to frame the question of where chaos exists within this structure.

The fact that quantum and classical mechanics are fundamentally incompatible in many ways, yet the macroscopic world is well-described by classical dynamics has puzzled physicists ever since the laying of the foundations of 
quantum theory. It is fair to say that not everyone is satisfied with the state of affairs - including many seasoned practitioners of quantum mechanics.

Of course, the notion of measurement in quantum mechanics - the denial of reality to system properties unless they are measured - is such a revolutionary concept that it engenders much more unease [8], even today. The problem is that, were quantum mechanics the final theory, it could deny reality to the measurement results themselves unless they were observed by another system and so on, ad infinitum. In order to "solve" the "measurement problem," it originally appeared impossible to think of quantum mechanics as a fundamental theory without relying on the existence of a classical world-view within which to embed it [9]. Although we still cannot dispel the unease invoked by the measurement problem, it is important to stress that the quantum-classical transition can be understood independently. This transition should not be confused with the measurement problem.

A partial understanding of the classical limit arises from the idea - familiar from nonequilibrium statistical mechanics - that weak interactions of a system with an environment are universal [10]. These interactions can effectively suppress certain nonclassical terms in the quantum evolution [11]. However, at best they only allow for the emergence of a classical probabilistic evolution and it can be shown that the mere existence of such interactions is insufficient to yield classical evolution in all cases [12]. Finally, this picture alone cannot explain the results of actual measurements where information can be continuously extracted from the environment and used to define operational notions of a trajectory. We now go in to these questions in more detail.

\section{Isolated and Open Evolution}

Suppose we are given an arbitrary system Hamiltonian $H(x, p)$ in terms of the dynamical variables $x$ and $p$; we will be more specific regarding the precise meaning of $x$ and $p$ as position and momentum later. The Hamiltonian is the generator of time evolution for the physical system state, provided there is no coupling to an environment or measurement device. In the classical case, we specify the initial state by a positive phase space distribution function $f_{C l}(x, p)$; in the quantum case, by the (position-representation) positive semidefinite density matrix $\rho\left(x_{1}, x_{2}\right)$ or, completely equivalently, by the corresponding Wigner distribution function $f_{W}(x, p)$ (not positive). The Wigner distribution $[13,14]$ is a "half-Fourier" transform of $\rho\left(x_{1}, x_{2}\right)$, defined as

$$
f_{W}(x, p)=\frac{1}{2 \pi \hbar} \int d \Delta \rho\left(x+\frac{1}{2} \Delta, x-\frac{1}{2} \Delta\right) \exp (-i p \Delta / \hbar),
$$

where $x \equiv\left(x_{1}+x_{2}\right) / 2$ and $\Delta \equiv x_{1}-x_{2}$.

The evolution of an isolated system is then given by the classical and quantum Liouville equations for the fine-grained distribution functions (i.e., the 
evolution is entropy-preserving):

$$
\begin{aligned}
\partial_{t} f_{C l}(x, p) & =-\left[\frac{p}{m} \partial_{x}-\partial_{x} V(x) \partial_{p}\right] f_{C l}(x, p), \\
\partial_{t} f_{W}(x, p)= & -\left[\frac{p}{m} \partial_{x}-\partial_{x} V(x) \partial_{p}\right] f_{W}(x, p) \\
& +\sum_{\lambda=1}^{\infty} \frac{(\hbar / 2 i)^{2 \lambda}}{(2 \lambda+1) !} \partial_{x}^{2 \lambda+1} V(x) \partial_{p}^{2 \lambda+1} f_{W}(x, p),
\end{aligned}
$$

where we have assumed for simplicity that the potential $V(x)$ can be Taylorexpanded; this does not alter the nature of any of the following arguments. Note that these evolutions are both linear in the respective distribution functions.

The limiting form $f_{C l}(x, p)=\delta(x-\bar{x}) \delta(p-\bar{p})$ is allowed classically, and, on substitution in Eqn. (2), yields the expected Newton's equations. These may then be interpreted as equations for the particle position and momentum, although we must emphasize that this identification is only formal at this stage. Quantum mechanically, this ultralocal limit is not permitted since $f_{W}(x, p)$ must be square-integrable, therefore - even formally - no direct particle interpretation can exist. In both cases, if one allows for initially localized distributions but which nevertheless have some finite width, it is easy to see that if $V(x)$ is nonlinear, quite generically the distribution will eventually spread over the allowed phase space and not remain localized.

As alluded to in the Introduction, the extension to open systems is conceptually trivial, but very difficult to implement in practice. To the original system Hamiltonian, we now add pieces representing the environment and the systemenvironment coupling. If the environment is in principle unobservable, then a (nonlocal in time) linear master equation for the system's reduced density matrix is - in theory - derivable by tracing over the environmental variables. In practice, tractable equations are impossible to obtain without drastic simplifying assumptions such as weak coupling, timescale separations, and simple forms for the environmental and coupling Hamiltonians. In any case, the important point to note is that the act of tracing over the environment does not change the linear nature of the equations. Generally speaking, master equations describing open evolution of coarse-grained distributions augment the RHS of Eqns. (2) and (3) with terms containing dissipation and diffusion kernels connected via generalized fluctuation-dissipation relations [15]. While the classical diffusion term vanishes in the limit of zero temperature for the environment, this is not true quantum mechanically due to the presence of zero-point fluctuations. 


\section{Continuous Measurement and Conditioned Evolution}

In contrast to classical theory, where measurement can be, in principle, a passive process, in quantum theory measurement creates an irreducible disturbance on the observed system (quantum "backaction"). This being so, if our aim is that measurement yield dynamical information - rather than strongly influence dynamics - the desired measurement process must yield a limited amount of information in a finite time. Hence, simple projective (von Neumann) measurements are clearly not appropriate because they yield complete information instantaneously via state projection. Nevertheless, this fundamental notion of measurement can be easily extended [5] to devise schemes that extract information continuously [16]. The basic idea is to have the system of interest interact weakly with another (e.g., atom interacting with an electromagnetic field) and make projective measurements on the auxiliary system (e.g., photon counting). Because of the weak interaction, the state of the auxiliary system gathers very little information regarding the system of interest, and therefore this system, in turn, is only perturbed slightly by the measurement backaction. Only a small component of the information gathered by the projective measurement of the auxiliary system relates to the system of interest, and a continuous limit of the measurement process can be taken.

In the continuous limit, the evolution of the system density matrix is fundamentally different from the equations discussed above for the case of open evolution. The master equation describing the evolution of the reduced density matrix conditioned on the results of the measurements contains a term that reflects the gain in information arising from the measurement record ("innovation" in the language of control theory). This term, arising from applying a continuous analog of Bayes' theorem, is intrinsically nonlinear in the distribution function. The coupling to an external probe (and the associated environment) will also cause effects very similar to the open evolution considered earlier, and there can once again be dissipation and diffusion terms in the evolution equations. The primary differences between the classical and quantum treatments, aside from the kinematic constraints on the distribution functions, are the following: (i) the (nonlocal in $p$ ) quantum evolution term in Eqn. (3), and (ii) an irreducible diffusion contribution due to quantum backaction reflecting the active nature of quantum measurements.

We now consider a simple model of position measurement to provide a measure of concreteness. In this model, we will assume that there are no environmental channels aside from those associated with the measurement. Suppose we have a single quantum degree of freedom, position in this case, undergoing a weak, ideal continuous measurement [16]. Here "ideal" refers to no loss of information during the measurement, i.e., a fine-grained evolution with no increase in entropy. Then, we have two coupled equations, one for the mea- 
surement record $y(t)$,

$$
d y=\langle x\rangle d t+\frac{1}{\sqrt{8 k}} d W
$$

where $d y$ is the infinitesimal change in the output of the measurement device in time $d t$, the parameter $k$ characterizes the rate at which the measurement extracts information about the observable, i.e., the strength of the measurement [17], and $d W$ is the Wiener increment describing driving by Gaussian white noise [18], the difference between the actually observed value and that expected. The other equation - the nonlinear stochastic master equation (SME) - specifies the resulting conditioned evolution of the system density matrix, given in the Wigner representation,

$$
\begin{aligned}
f_{W}(x, p, t+d t)= & {\left[1+d t\left[-\frac{p}{m} \partial_{x}+\partial_{x} V(x) \partial_{p}+D_{B A} \partial_{p}^{2}\right]\right.} \\
& \left.+d t \sum_{\lambda=1}^{\infty} \frac{(\hbar / 2 i)^{2 \lambda}}{(2 \lambda+1) !} \partial_{x}^{2 \lambda+1} V(x, t) \partial_{p}^{2 \lambda+1}\right] f_{W}(x, p, t) \\
& +d t \sqrt{8 k}(x-\langle x\rangle) f_{W}(x, p, t) d W
\end{aligned}
$$

where $D_{B A}=\hbar^{2} k$ is the diffusion coefficient arising from quantum backaction and the last (nonlinear) term represents the conditioning due to the measurement. In principle, there is also a (generalized) damping term [19], but if the measurement coupling is weak enough, it can be neglected. If we choose to average over all the measurement results, which is the same as ignoring them, then the conditioning term vanishes, but not the diffusion from the measurement backaction. Thus the resulting linear evolution of the coarsegrained quantum distribution is not the same as the linear fine-grained evolution (3), but yields a conventional open-system master equation. Moreover, for a given (coarse-grained) master equation, different underlying fine-grained SME's may exist, specifying different measurement possibilities.

In contrast to the quantum case, the corresponding (ideal) classical conditioned master equation [set $\hbar=0$ in Eqn. (5), holding $k$ fixed],

$$
\begin{aligned}
f_{C l}(x, p, t+d t)= & {\left[1-d t\left[\frac{p}{m} \partial_{x}-\partial_{x} V(x) \partial_{p}\right]\right] f_{C l}(x, p, t) } \\
& +d t \sqrt{8 k}(x-\langle x\rangle) f_{C l}(x, p, t) d W
\end{aligned}
$$

does not have the backaction term as these classical measurements are passive: averaging over all measurements simply gives back the Liouville equation (2), and there is no difference between the fine-grained and coarse-grained evolutions in this special case. [In general, classical diffusion terms from ordinary open evolution can also coexist, as in the a posteriori evolution specified by the Kushner-Stratonovich equation [20], of which Eqn. (6) is a special case.] 
As a final point, we delay our discussion of how the classical trajectory limit is incorporated in Eqn. (6), i.e., the precise sense in which the "the position of a particle is what a position-detector detects," to the next section.

\section{QCT: The Quantum-Classical Transition}

If quantum mechanics is really the fundamental theory of our world, then an effectively classical description of macroscopic systems must emerge from it - the so-called quantum-classical transition (QCT). It turns out that this issue is inextricably connected with the question of the physical meaning of dynamical nonlinearity discussed above. Having written down the relevant evolution equations, we now analyze two notions of the QCT and how they emerge from the equations.

Quantum mechanics is intrinsically probabilistic, but classical theory - as shown above by the existence of the delta-function limit for the classical distribution function - is not. Since Newton's equations provide an excellent description of observed classical systems, including chaotic systems, it is crucial to establish how such a localized, or trajectory, description can arise quantum mechanically. We will call this the strong form of the QCT. Of course, in many situations, only a statistical description is possible even classically, and here we demand only the agreement of quantum and classical distributions and the associated dynamical averages. This defines the weak form of the QCT.

It is clear that if the strong form of the QCT holds, then, via trivial coarsegraining, the weak form follows automatically. The reverse is not true, however: results from a coarse-grained analysis cannot be applied to the finegrained situation. Moreover, the violation of the conditions necessary to establish the strong form of the QCT need not prevent the existence of a weak QCT. We now discuss and establish the conditions under which these transitions occur. Since the strong form of the QCT requires treating the localized limit, a cumulant expansion for the distribution function immediately suggests itself, whereas, for the more nonlocal issues relevant to the weak form of the QCT, a semiclassical analysis turns out to be natural.

\subsection{Strong Form of the QCT: Chaos in the Classical Limit}

It is easy to see that the strong form of the QCT is impossible to obtain from either the isolated or open evolution equations for the density matrix or Wigner function. As mentioned already, for a generic dynamical system, a localized initial distribution tends to distribute itself over phase space - and then continue to evolve - either in complicated ways (isolated system) or asymptote to an equilibrium state (open system), whether classically or quantum mechanically. In the case of conditioned evolution, however, the distribution can be localized due to the information gained from the measurement, and evolve in a quite 
different manner. In order to quantify how this happens, let us first apply a cumulant expansion to the (fine-grained) conditioned classical evolution (6). This results in the following equations for the centroids $(\bar{x} \equiv\langle x\rangle, \bar{p} \equiv\langle p\rangle)$,

$$
\begin{aligned}
d \bar{x} & =\frac{\bar{p}}{m} d t+\sqrt{8 k} C_{x x} d W, \\
d \bar{p} & =\langle F(x)\rangle d t+\sqrt{8 k} C_{x p} d W,
\end{aligned}
$$

where

$$
\begin{aligned}
F(x) & =-\partial_{x} V(x), \\
C_{A B} & =\frac{1}{2}(\langle A B\rangle+\langle B A\rangle-2\langle A\rangle\langle B\rangle),
\end{aligned}
$$

along with a hierarchy of coupled equations for the time-evolution of the higher cumulants. These equations are the continuous measurement, real-world, ana$\log$ of the formal ultralocal Newtonian limit of the distribution function in the classical Liouville equation (2). Whereas Eqns. (7) always apply, our aim is to determine the conditions under which the cumulant expansion effectively truncates and brings their solution very close to that of Newton's equations. This will be true provided the noise terms are small (in an average sense) and the force term is localized, i.e., $\langle F(x)\rangle=F(\bar{x})+\cdots$, the corrections being small. The required analysis involves higher cumulants and has been carried out elsewhere [21]. It turns out that the distribution is localized provided

$$
8 k \gg \sqrt{\frac{\left(\partial_{x}^{2} F\right)^{2}\left|\partial_{x} F\right|}{2 m F^{2}}}
$$

and the motion of the centroid will effectively define a smooth classical trajectory - the low-noise condition - as long as

$$
k \gg \frac{2\left|\partial_{x} F\right|}{S}
$$

where $S$ is the action scale of the system. Note that this condition does not bound the measurement strength: classically we can always extract as much information as needed - at least in principle - to gain the trajectory limit. This, then, is the "realistic" derivation of Newton's equations.

We now turn to the quantum version of these results. In this case, the analogous cumulant expansion gives exactly the same equations for the centroids as above, while the equations for the higher cumulants are different. (The evolution of classical and quantum averages is the same to Gaussian order, with the first differences arising at the next order [22].) We can again investigate whether a trajectory limit exists. Localization holds in the weakly nonlinear 
case if the classical condition above is satisfied. In the case of strong nonlinearity, the inequality becomes [21]

$$
8 k \gg \frac{\left(\partial_{x}^{2} F\right)^{2} \hbar}{4 m F^{2}} .
$$

Because of the backaction, the low-noise condition is implemented in the quantum case by a double-sided inequality:

$$
\frac{2\left|\partial_{x} F\right|}{s} \ll \hbar k \ll \frac{\left|\partial_{x} F\right| s}{4},
$$

where the action is measured in units of $\hbar, s$ being dimensionless. The left inequality is the same as the classical one discussed above, however the right inequality is essentially quantum mechanical. The measurement strength cannot be made arbitrarily large as the backaction will result in too large a noise in the equations for the centroids. As the action $s$ is made larger, both inequalities are satisfied for an ever wider range of values of $k$. For sufficiently large $s$, the actual value of $k$ becomes irrelevant and the dynamics becomes effectively classical.

To recapitulate, for continuously measured quantum systems, trajectories that emerge in the macroscopic limit follow Newton's equations, and hence can be chaotic as shown elsewhere [21]. Thus, as speculated in a prescient paper by Chirikov [23], measurement indeed provides the missing link between "quantum" and "chaos," at least in the classical limit.

Finally, in experiments one usually considers the measurement record itself rather than the estimated state of the system as we have discussed so far. As measurement introduces a white noise, it is important to investigate the condition under which the record tracks the estimate faithfully. If $\Delta t$ is the time over which the continuous measurement is averaged to obtain the record (this averaging being a necessary part of any finite-bandwidth experiment), and we allow ourselves a maximum of $\Delta x$ as the position noise, it is easy to see that the measurement strength needs to satisfy [21]

$$
8 k>\frac{1}{\Delta t(\Delta x)^{2}}
$$

To demonstrate these results for a concrete example, we revisit the results of Ref. [21] for a driven, Duffing oscillator, with system Hamiltonian

$$
H=P^{2} / 2 m+B x^{4}-A x^{2}+\Lambda x \cos (\omega t),
$$

with $m=1, B=0.5, A=10, \Lambda=10, \omega=6.07$. This Hamiltonian has been used before in studies of quantum chaos [24] and quantum decoherence [25] 

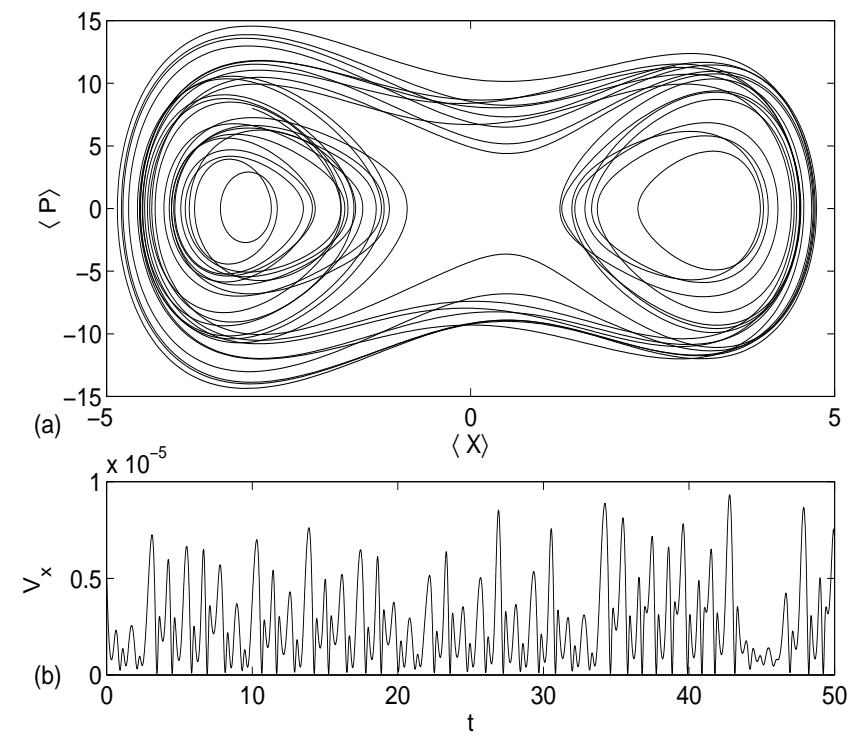

Figure 1. (a) The quantum trajectory in phase space for a continuously measured Duffing oscillator [21], with $\hbar=10^{-5}$ and $k=10^{5}$. (b) The position variance, $V_{x}$, as a function of time. Note the smallness of the scale on the y-axis!

and, in the parameter regime used, a substantial area of the accessible phase space is stochastic.

Numerical calculations at various values of $\hbar$ confirm that as $\hbar$ is reduced, both the steady-state variance, and the resulting noise (for optimal measurement strengths) are reduced, as expected. As the dynamical time scale of this problem is $1-0.1$ (in units of the driving period), the continuous observation record was averaged over a period of 0.01 . Similarly, as the range of the motion covers distances of $O(10)$, we demand that the position be tracked to an accuracy of 0.01 to define an effective "trajectory." To satisfy this, we need to have $k \sim O\left(10^{5}\right)$ or larger [Cf. Eqn. (13)]. In our example, we choose the energy to be $O\left(10^{2}\right)$, the corresponding typical action turns out to be $O(10)$, and the typical nonlinearity makes the RHS of Eqn. 9, $O(1)$. We see that a choice of $\hbar=10^{-5}$ and $k=10^{5}$, satisfies all the constraints for a classical motion. In Fig. 1 we demonstrate that in this regime, localization is maintained along with low levels of trajectory noise. Fig. 1(a) shows a typical phase space trajectory, with the position variance during the evolution, $V_{x} \equiv(\Delta x)^{2}$, plotted in Fig. 1(b). We find that the width $\Delta x$ is always bounded by $3.4 \times 10^{-3}$. Furthermore, as is immediately evident from the smoothness of the trajectory in Fig. 1(a), the noise is also negligible on these scales. Additionally, one can verify that the quantum trajectory evolution and that given by a classical trajec- 
tory with an equivalent noise are essentially identical - and chaotic - yielding a Lyapunov exponent of 0.57 . We return to discuss the Lyapunov exponent later below.

\subsection{Chaos and the Weak Form of the QCT}

The weak form of the QCT utilizes the coarse-grained distribution function (averaging over all measurements), whereas the strong form refers to the fine-grained distribution for a single measurement realization. It is important to reiterate that nonexistence of the strong form of the QCT does not influence the existence of the weak form of the QCT: It does not matter if the distribution is too wide, as long as the classical and quantum distributions agree, and, even if the backaction noise is large, the coarse-grained distribution can remain smooth and the weak quantum-classical correspondence still exist. Consequently, this correspondence has to be approached in a different manner. In fact, the weak version is just another way to state the conventional decoherence idea [11]; however, as discussed elsewhere [12], mere suppression of quantum interference does not guarantee the QCT even in the weak form.

We now focus on a semiclassical analysis of the weak QCT for bounded, classically chaotic open systems [26]. This analysis is best regarded as a regularization of the singular $\hbar \rightarrow 0$ limit via the environmental interaction. This is distinct from the state localization characteristic of the strong form of the QCT. Given a small, but finite, value of $\hbar$, the aim is to establish the existence of a timescale beyond which the dynamics of open quantum and classical systems becomes statistically equivalent if the environmental interaction is sufficiently strong.

It has been demonstrated [26] that, for a bounded open system with a classically chaotic Hamiltonian, the weak form of the QCT is achieved by two parallel processes, both relying essentially on the existence of environmental diffusion. First, the semiclassical approximation for quantum dynamics, which breaks down for classically chaotic systems due to overwhelming nonlocal interference, is recovered as the environmental interaction filters these effects. Second, environmental noise restricts the foliation of the unstable manifold, the set of points which approach a hyperbolic point in reverse time, allowing the semiclassical wavefunction to track this modified classical geometry. In this way, the noise prevents classical chaos from breaking the semiclassical approximation as $\hbar \rightarrow 0$, and thus regularizes this limit. Note that this approach explicitly incorporates both the stretching and folding typical of hyperbolic regions as well as the role of the environment as a filter on a phase-space quantum distribution.

We begin with a simple model of a quantum system weakly coupled to the environment so as to maintain complete positivity for the subsystem density 
matrix, $\rho(t)$, while subjecting it to a, time-local, unitarity-breaking interaction. These conditions mathematically constrain the master equation to be of the so-called Lindblad form [27]. If this environmental interaction couples to the position, as is often the case, the master equation takes the form:

$$
\frac{\partial f_{w}}{\partial t}=L_{c l} f_{w}+L_{q} f_{w}+D \frac{\partial^{2} f_{w}}{\partial p^{2}}
$$

where $L_{c l}$, the classical Liouville operator, and $L_{q}$, the quantum correction, can be easily identified from Eqn. (3). We note in passing that while the sum of $L_{c l}$ and $L_{q}$ is clearly unitary, individually the operators are not unitary [12]. In this simple master equation, we have neglected the dissipative environmental channel and kept the diffusive channel for two reasons: (i) the coupling to the environment is always assumed to be weak and the dissipative timescales are, hence, very long, longer than the dynamical timescales of interest, (ii) the weak form of the QCT arises only from the diffusive channel, hence, dissipative effects are not of interest here.

When $L_{q}=0$, this equation reverts to the classical Fokker-Planck equation. It is important to keep in mind that the specific form of the diffusion coefficient depends strongly on the physical situation envisaged. Thus, if the master equation describes a weakly coupled, high temperature environment, $D=2 m \gamma k_{B} T$ ( $\gamma$ is the damping coefficient) [28], whereas for a weak, continuous measurement of position, the diffusion due to quantum backaction is $D=\hbar^{2} k$ [16]. The discussion below holds for all of these cases.

Once the QCT occurs, the effects of $L_{q}$ in the evolution specified by Eqn. (15) are subdominant. Therefore, to understand how environmental noise acts in this limit, it suffices to consider the behavior of the corresponding classical Fokker-Planck equation. To do this, it is convenient to examine the underlying Langevin equations for noisy trajectories that unravel the evolution of the classical distribution function when $L_{q}=0$. These are given by

$$
\begin{aligned}
d q & =\frac{p}{m} d t \\
d p & =f(q) d t+\sqrt{2 D} d W
\end{aligned}
$$

Using weak-noise perturbation theory, one can perform an expansion about a hyperbolic fixed point and in this way obtain the spreading of the position and momentum due to the diffusion. As a trajectory evolves, it simultaneously smoothes over a transverse width in phase space of size $\sqrt{D t /(m \lambda)}$ where $\lambda$ is the local Lyapunov exponent [26].

The smoothing implies a termination in the development of new phase space structures at some finite time $t^{*}$, whose scaling behavior can be determined. (Caveat: this need not be true in a non-compact phase space.) The average motion of a trajectory is identical to its deterministic motion, so that at time $t$, 
if the initial length in phase space is $u_{0}$ ( $u$ has units of square-root of phasespace area), its current length will be approximately $u_{0} e^{\bar{\lambda} t}$ as its forward time evolution will be dominated by its component in the unstable direction. Here $\bar{\lambda}$ is the time-averaged positive Lyapunov exponent. If the region is bounded within a phase space area $A$, the typical distance between neighboring folds of the trajectory is given by

$$
l(t) \approx \frac{A}{u_{0}} e^{-\bar{\lambda} t}
$$

where $l(t)$ still carries the units of the square root of phase space area. However, since phase structures can only be known to within the width specified above, the time at which any new structure will be smoothed over is defined by

$$
l\left(t^{*}\right) \approx \sqrt{\frac{D t^{*}}{m \bar{\lambda}}} .
$$

The above two equations can be used to determine $t^{*}$, which only weakly depends on $D$ and the prefactor in Eqn. (17). Due to the smoothing, one does not see an ergodic phase space region, but one in which the large, short-time features that develop prior to $t^{*}$ are pronounced and the small, long-time features that develop later are smoothed over by the averaging process. Therefore, to approximate noisy classical dynamics, a quantum system need not track all of the fine scale structures, but only the larger features that develop before the production of small scale structures terminates.

To establish the conditions under which quantum dynamics can track this modified phase space geometry, a semiclassical analysis can be performed. In the Wigner function formalism, the breakdown of the semiclassical approximation for chaotic systems can be associated with an appealing geometric picture $[29,14]$ based on a uniform approximation in phase space - the Berry construction. We now use this construction to understand how quantum interference in phase space is smoothed over by the diffusion associated with environmental coupling.

A general mixed state is an incoherent superposition of pure state Wigner functions, where an individual semiclassical pure state Wigner function can be formed by substituting the Van-Vleck semiclassical wavefunction in Eqn. (1). If we allow $q$ to be perturbed by noise we can rewrite the classical action [30]

$$
S(q, t) \approx S\left(q_{C}, t\right)-\sqrt{2 D} \int_{0}^{t} d t \xi(t) q_{C}(t) .
$$

Following Berry [29], we now rewrite the action for the $i$ th solution to the Hamilton-Jacobi equation as

$$
S_{i}\left(q_{C}, t\right)=\int_{q_{C}(0)}^{q_{C}(t)} d q^{\prime} p_{i}\left(q^{\prime}, t\right)-\int_{0}^{t} d t^{\prime} H\left(q_{C}(0), p_{i}\left(q_{C}(0), t^{\prime}\right)\right.
$$




$$
\equiv \int_{0}^{t} d t^{\prime} \mathcal{H}_{i}\left(t^{\prime}\right)
$$

where $p_{i}(q, t)$ is the $i$ th branch of the momentum curve for a given $q$. If we average over all noisy realizations, after separating the contributions from identical branches, the following suggestive expression for the noise averaged semiclassical Wigner function obtains:

$$
\begin{aligned}
& \frac{1}{2 \pi \hbar} \int_{-\infty}^{\infty} d X \exp \left(-\frac{D t X^{2}}{2 \hbar^{2}}\right)\left(\sum_{i} \mathcal{J}_{i i} \times\right. \\
& \exp \left[\frac{i}{\hbar}\left\{\int_{\bar{q}_{-}}^{\bar{q}_{+}} d q^{\prime} p_{i}\left(q^{\prime}, t\right)-p X\right\}\right]+ \\
& 2 i \sum_{i<j} \mathcal{J}_{i j} \sin \left[\frac { 1 } { \hbar } \left\{\int_{q_{C}(0)}^{\bar{q}_{+}} d q^{\prime} p_{i}\left(q^{\prime}, t\right)-\int_{q_{C}(0)}^{\bar{q}_{-}} d q^{\prime} p_{j}\left(q^{\prime}, t\right)\right.\right. \\
& \left.\left.\left.-\int_{0}^{t} d t^{\prime}\left(\mathcal{H}_{i}-\mathcal{H}_{j}\right)+\phi_{i}-\phi_{j}\right\}\right]\right) \\
& \mathcal{J}_{i j} \equiv \frac{C_{i}\left(\bar{q}_{+}, t\right) C_{j}\left(\bar{q}_{-}, t\right)}{\sqrt{\left|J_{i}\left(\bar{q}_{+}, t\right)\right|\left|J_{j}\left(\bar{q}_{-}, t\right)\right|}}
\end{aligned}
$$

for Jacobian determinant $J_{i}(q, t)$ and transport coefficient $C_{i}(q, t) ; \bar{q}_{ \pm} \equiv q \pm \frac{X}{2}$ and $\phi_{i}=\pi \nu_{i}$, where $\nu_{i}$ is the $i$ th Maslov index [31].

The dominant contributions to the integrals can be analyzed in the stationary phase approximation [32]. If $D=0$, these would contribute phase coherences at values of $X$ that satisfy $p_{i}(q+X / 2, t)+p_{i}(q-X / 2, t)-2 p X=0$ for the first term in the sum and $p_{i}(q+X / 2, t)+p_{j}(q-X / 2, t)-2 p X=0$ for the second term, the former being the famous Berry midpoint rule. For a chaotic system, Berry argued that, due to the proliferation of momentum branches, $p_{i}(q, t)$, arising from the infinite number of foldings of a bounded chaotic curve as $t \rightarrow \infty$, a semiclassical approximation would eventually fail, since the interference fringes stemming from a given $p_{i}$ could not be distinguished after a certain time from those emanating from the many neighboring branches [32]. While the precise value of this time has since been challenged numerically, the essential nature of this physical argument has remained valid [33].

In the present case, however, the presence of noise acts as a dynamical Gaussian filter, damping contributions for any solutions to the above equation which are greater than $X \approx \hbar / \sqrt{D t}$. In other words, noise dynamically filters the long "De Broglie" wavelength contributions to the semiclassical integral, the very sort of contributions which generally invalidate such an approximation. If we rescale the above result and combine it with our understanding of how noise effects classical phase space structures, we can qualitatively estimate whether or not a semiclassical picture is a valid approximation to the dynamics. As 
already discussed, $t^{*}$ is the time when the formation of new classical structures ceases and $l\left(t^{*}\right)$ is the associated scale over which classical structures are averaged. The key requirement is then that the semiclassical phase filters contributions of size

$$
\frac{\sqrt{\bar{\lambda} m} \hbar}{\sqrt{D t^{*}}} \lesssim l\left(t^{*}\right)
$$

In other words, for a given branch, the phases with associated wavelengths long enough to interfere with contributions from neighboring branches are strongly damped, and the intuitive semiclassical picture of classical phase-space distributions decorated by local interference fringes recovered.

The weak form of the QCT is completed when the inequality (23) is satisfied. Substituting the scale of classical smoothing (18) in this inequality, we find

$$
D t^{*} \gtrsim \bar{\lambda} m \hbar \text {. }
$$

[Note that the purely classical quantity $t^{*}$ is first independently determined by solving Eqn. (18) and then compared to the right side of the above equation.] While the left hand side of the inequality contains the mutually dependent $t^{*}$ and $D$, the right hand side depends only on fixed properties of the system and $\hbar$. This condition, therefore, defines a threshold at which the semiclassical approximation becomes stable and that may be set in terms of either $D$ or $t^{*}$. Once the threshold is met, $t^{*}$ becomes the time beyond which the semiclassical description is valid. The semiclassical nature of this condition becomes more evident on defining $S=l\left(t^{*}\right)^{2}$ which, given that $l^{2}$ is an areal scale in phase space for the diffusion averaged dynamics, has dimensions of action. A physical interpretation is more apparent on rewriting (24) as $S=l\left(t^{*}\right)^{2} \gtrsim \hbar$, which is readily identified as the usual condition for the validity of a semiclassical analysis.

The weak form of the QCT can also be demonstrated using the Duffing example [26]. The dynamical evolution of the bounded motion is dominated by the homoclinic tangle of a single hyperbolic fixed point. As a result, the long-time chaotic evolution can be completely characterized by the unstable manifold associated with that fixed point [34]. The value of $\hbar$ is now set to $\hbar=0.1$, significantly larger than when studying the strong form of the QCT.

The evolution of the corresponding distributions was numerically calculated for both the classical and quantum master equations. Fig. 2 shows sectional cuts at $p=0$ of the quantum and classical phase space distribution functions for three different values of the diffusion coefficient, $D=10^{-5}, 10^{-3}, 10^{-2}$, after time $T=149$ evolution periods. As already mentioned, $t^{*}$ varies slowly with $D$, and in the three cases shown, $t^{*}$ ranges only from $\sim 20-14$ (note that $t^{*} \ll T$ ). It is easy to check that the inequality (24) is strongly violated for $D=10^{-5}$, mildly violated for $D=10^{-3}$, and approximately satisfied for 


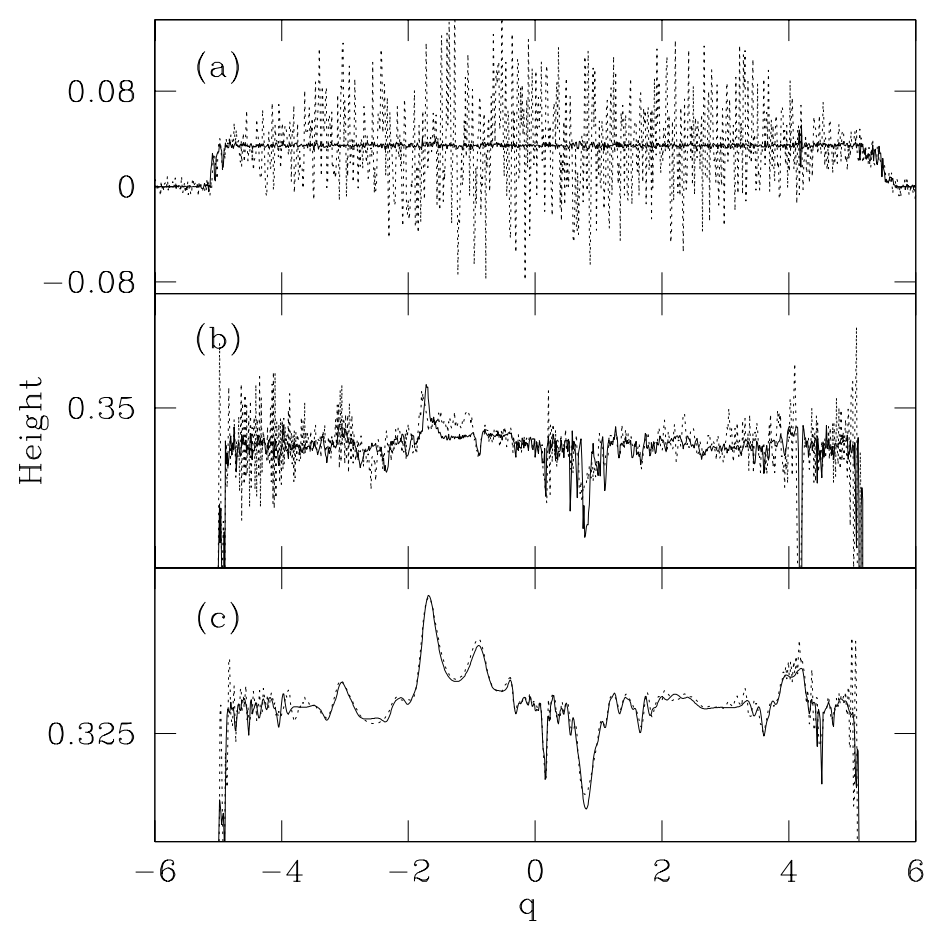

Figure 2. The weak form of the QCT for a driven Duffing oscillator [26]: Sectional cuts of Wigner functions (dashed lines) and classical distributions (solid lines), after 149 drive periods, taken at $p=0$ for diffusion coefficient values (a) $D=10^{-5}$; (b) $D=10^{-3}$; (c) $D=10^{-2}$. Other parameter values are stated in the text; the height is specified in scaled units.

$D=10^{-2}$. For $D=10^{-5}$, the classical and quantum sections show no similarities, as expected. The quantum Wigner function also shows large negative regions, reflecting strong quantum interference. On increasing $D$ to $10^{-3}$ the magnitude of quantum coherence decreases dramatically and the classical and quantum slices have the same average value, as well as specific agreement on some large scale features. The two disagree, as expected, on the small scale structures. This indicates that, while the quantum and classical distributions do not exactly match, the Wigner function has now become sensitive to the larger features of the noise averaged classical distribution function, indicative of the transition to a semiclassical regime. At $D=10^{-2}$, there is near perfect agreement between classical and quantum distribution functions, save on the smallest scales. When $D$ is of order unity, the inequalities enforcing the strong QCT at the level of individual trajectories [21] are satisfied and the agreement 
is essentially exact. However, as indicated by Fig. 2(c), detailed agreement for quantum and classical distribution functions can begin at much smaller values of the diffusion constant.

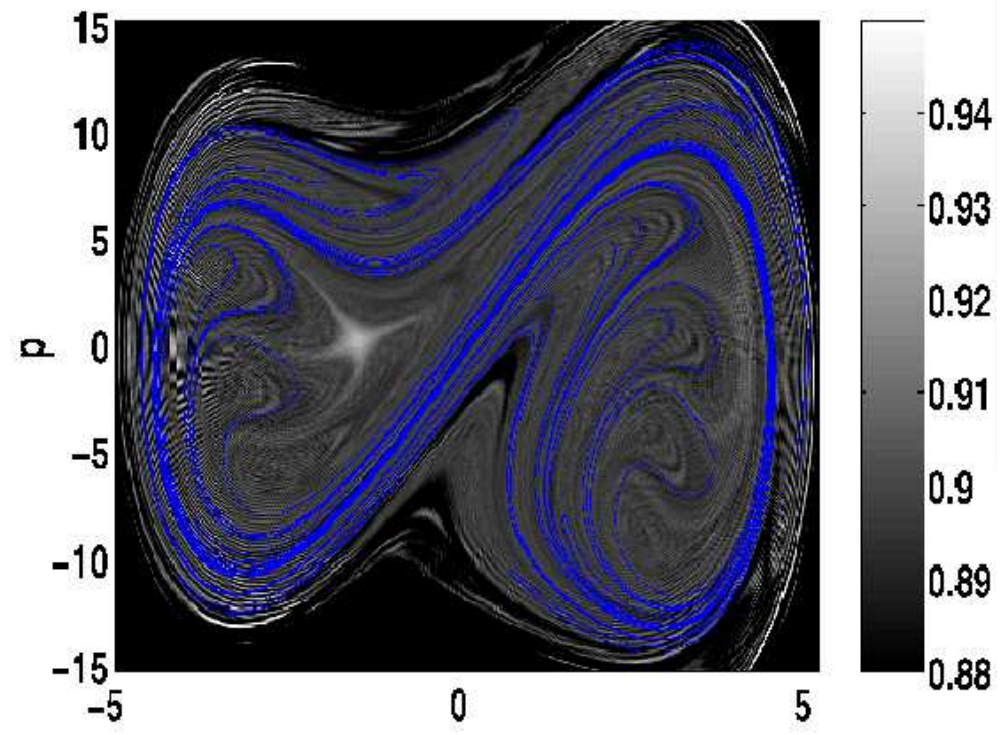

Figure 3. Phase space rendering of the Wigner function at time $t=149$ periods of driving [26]. The early time part of the unstable manifold associated with the noise-free dynamics is shown in blue. The value of $D=10^{-3}$ is not sufficient to wipe out all the quantum interference which, as expected, is most prominent near turns in the manifold.

For more detailed evidence that, at $D=10^{-3}$, one is entering a semiclassical regime, in Fig. 3 we superimpose an image of the large scale features of the classical unstable manifold on top of the full quantum Wigner distribution at $D=10^{-3}$ after 149 drive periods [case (b) of Fig. 2]. The quantum phase space clearly exhibits local interference fringes around the large lobe-like structures associated with the short-time evolution of the unstable manifold. The appearance of local fringing about classical structures is direct evidence of a semiclassical evolution, where interference effects appear locally around the backbone of a classical evolution. This is in sharp contrast to the global diffraction pattern seen for $D=0$, where the contributions from individual curves cannot be distinguished, suppressing the appearance of any classical structure [25]. 


\section{Chaos in Quantum Mechanics}

At this point, our analysis of measured quantum dynamical systems may be said to have harmonized quantum and classical mechanics in the sense that the strong and weak forms of the QCT have appeared naturally. While this is certainly pleasing, we wish to go further and ask whether the formalism can be tested by making predictions that are experimentally verifiable and depend uniquely on the nonlinear nature of the conditioned evolution. One very interesting idea is the real-time control of quantum systems using state-estimation as pioneered by Belavkin [35] or direct feedback of the measured classical current [36]. Although quantum feedback control applications [37] have their own importance, we now return to the original burning question: Is there chaos in quantum mechanics?

In a limiting case, the answer is clearly in the affirmative. We have already shown that quantum distributions, provided certain conditions are met, can evolve while staying localized and be only very weakly perturbed by noise. In the classical limiting case, we recover localized classical trajectories, and these can certainly be chaotic. But what if these conditions are not satisfied?

This is the question addressed and answered in Ref. [38]. By defining and computing the Lyapunov exponent for an observed quantum system deep in the quantum regime, we were able to show that the system dynamics is chaotic. Further, the Lyapunov exponent is not the same as that of the classical dynamics that emerges in the classical limit. Since the quantum system in the absence of measurement is not chaotic, this chaos must emerge as the strength of the measurement is increased, and we examined the nature of this emergence.

To do this, we must first make certain that we can quantify the existence of chaos in a robust way. The rigorous quantifier of chaos in a dynamical system is the maximal Lyapunov exponent [39]. The exponent yields the (asymptotic) rate of exponential divergence of two trajectories which start from neighboring points in phase space, in the limit in which they evolve to infinity, and the neighboring points stay infinitesimally close. The maximal Lyapunov exponent characterizes the sensitivity of the system evolution to changes in the initial condition: if the exponent is positive, then the system is exponentially sensitive to initial conditions, and is said to be chaotic. We now discuss how this notion can be applied to observation-conditioned evolution of quantum expectation values.

A single quantum mechanical particle is in principle an infinite dimensional system. However, for the purpose of defining an observationally relevant Lyapunov exponent, it is sufficient to use a single projected data stream: let us consider the expectation value of the position, $\langle x(t)\rangle$. The important quantity is thus the divergence, $\Delta(t)=\left|\langle x(t)\rangle-\left\langle x_{\text {fid }}(t)\right\rangle\right|$, between a fiducial trajectory and a second trajectory infinitesimally close to it. It is important to keep 
in mind that the system is driven by noise. Since we wish to examine the sensitivity of the system to changes in the initial conditions, and not to changes in the noise, we must hold the noise realization fixed when calculating the divergence. The Lyapunov exponent is thus

$$
\lambda \equiv \lim _{t \rightarrow \infty} \lim _{\Delta_{s}(0) \rightarrow 0} \frac{\ln \Delta_{s}(t)}{t} \equiv \lim _{t \rightarrow \infty} \lambda_{s}(t)
$$

where the subscript $s$ denotes the noise realization. This definition is the obvious generalization of the conventional ODE definition to dynamical averages, where the noise is treated as a drive on the system. Indeed, under the conditions when (noisy) classical motion emerges, and thus when localization holds, it reduces to the conventional definition, and yields the correct classical Lyapunov exponent. To combat slow convergence, we measure the Lyapunov exponent by averaging over an ensemble of finite-time exponents $\lambda_{s}(t)$ instead of taking the asymptotic long-time limit for a single trajectory.

A key result now follows: In unobserved, i.e., isolated quantum dynamical systems, it is possible to prove, by employing unitarity and the Schwarz inequality, that $\lambda$ vanishes; the finite-time exponent, $\lambda(t)$, decays away as $1 / t$ [40]. From the Kosloff-Rice theorem we know, of course, that the Lyapunov exponent must be zero, since the overall evolution is integrable, but this result gives us a quantitative statement regarding the decay of the exponent. It turns out that this particular result applies also to the evolution of averages in isolated classical systems and, in this sense, is more general than Kosloff-Rice. As we have emphasized earlier, once measurement is included, the evolution becomes nonlinear and the Lyapunov exponent need not vanish classically or quantum mechanically.

As a particular system of interest, we turn once again to the Duffing oscillator, this time with $\hbar=10^{-2}$, which is small enough so that the system makes a transition to classical dynamics when the measurement is sufficiently strong. As we increase the measurement strength, we can examine the transformation from essentially isolated quantum evolution all the way to the known chaos of the classical Duffing oscillator. To examine the emergence of chaos, in Ref. [38] we solved for the evolution of the system for $k=5 \times 10^{-4}, 10^{-3}, 0.01,0.1,1,10$. When $k \leq 0.01$, the distribution is spread over the entire accessible region, and Ehrenfest's theorem is not satisfied. Conversely, for $k=10$, the distribution is well-localized (Fig. 4), and Ehrenfest's theorem holds throughout the evolution. Since the backaction noise, characterized by the momentum diffusion coefficient, $D=\hbar^{2} k$, remains small, at this value of $k$ the motion is that of the classical system, to a very good approximation.

Stroboscopic maps help reveal the global structural transformation in phase space in going from quantum to classical dynamics (Fig. 5). The maps consist 


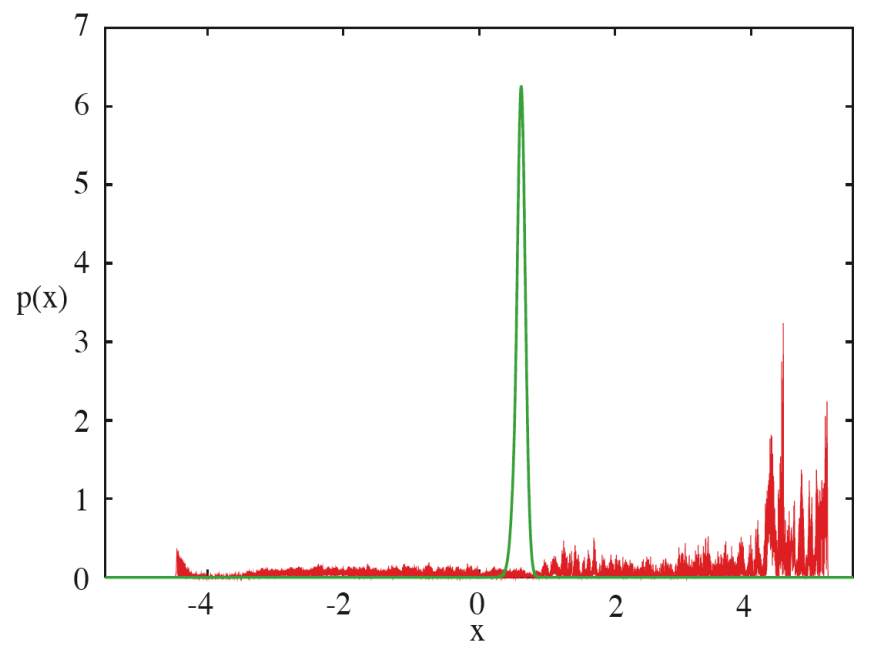

Figure 4. Position distribution for the Duffing oscillator with measurement strengths $k=$ 0.01 (red) and $k=10$ (green), demonstrating measurement-induced localization $(k=10)$ as the measurement coupling is increased [38]. The momentum distribution behaves similarly.

of points through which the system passes at time intervals separated by the period of the driving force. For very small $k,\langle x\rangle$ and $\langle p\rangle$ are largely confined to a region in the center of phase space. Somewhat remarkably, at $k=0.01$, although the system is largely delocalized, as shown in Fig. 4, nontrivial structure appears, with considerable time being spent in certain outer regions. By $k=1$ the localized regions have formed into narrower and sharper swirling coherent structures. At $k=10$ the swirls disappear, and we retrieve the uniform chaotic sea of the classical map (the small "holes" are periodic islands). The swirls in fact correspond to the unstable manifolds of the classical motion. Classically, these manifolds are only visible at short times, as continual and repeated folding eventually washes out any structure in the midst of a uniform tangle. In the quantum regime, however, the weakness of the measurement, with its inability to crystallize the fine structure, has allowed them to survive: we emphasize that the maps result from long-time integration, and are therefore essentially time-invariant.

To calculate the Lyapunov exponent we implemented a numerical version of the classical linearization technique [41], suitably generalized to quantum trajectories. The method was tested on a classical noisy system with comparison against results obtained from solving the exact equations for the Lyapunov exponents [42]. The calculation is very numerically intensive, as it involves integrating the stochastic Schrodinger equation equivalent to the SME (5) over 

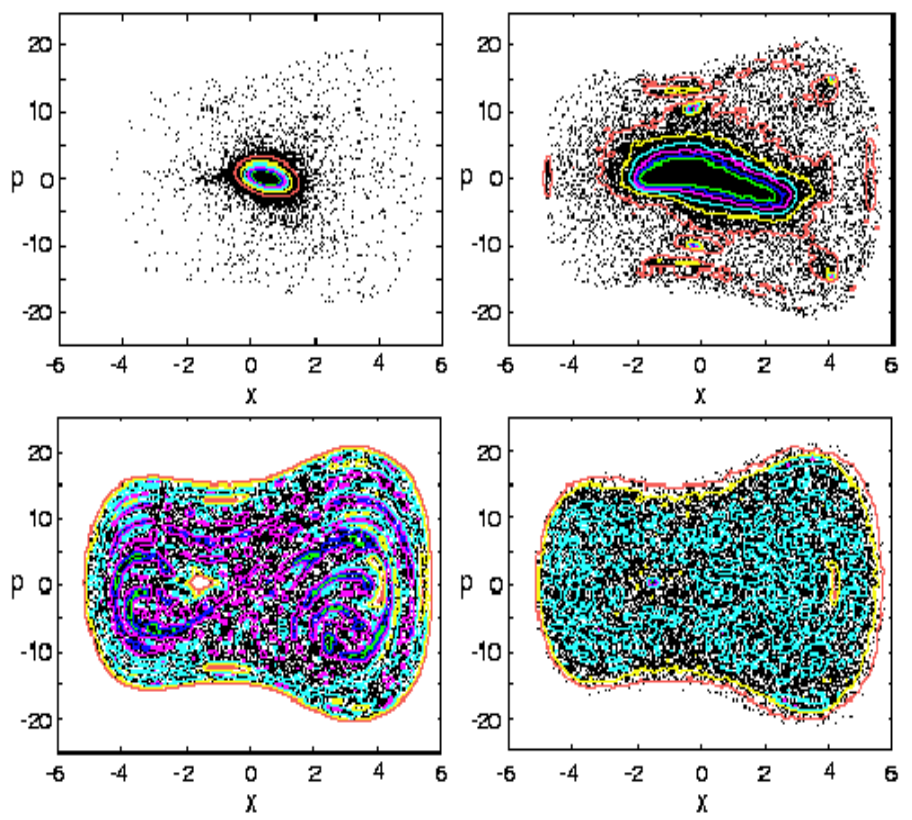

Figure 5. Phase space stroboscopic maps [38] for the observed Duffing oscillator for 4 different measurement strengths, $k=5 \times 10^{-4}, 0.01$ (top), and 1, 10 (bottom). Contour lines are superimposed to provide a measure of local point density at relative density levels of $0.05,0.15,0.25,0.35,0.45$, and 0.55 .

thousands of driving periods, and averaging over many noise realizations; parallel supercomputers were invaluable for this task.

The computations show that as $t$ is increased, for nonzero $k$, the value obtained for $\lambda(t)$ falls as $1 / t$, following the behavior expected for $k=0$, until a point at which an asymptotic regime takes over, stabilizing at a finite value of the Lyapunov exponent as $t \rightarrow \infty$. This behavior is shown in Fig. 6 for three values of $k$. The Lyapunov exponent as a function of $k$ is shown in Fig. 7. The exponent increases over two orders of magnitude in an approximately powerlaw fashion as $k$ is varied from $5 \times 10^{-4}$ to 10 , before settling to the classical value, $\lambda_{C l}=0.57$. The results in Figs. 6 and 7 show clearly that chaos emerges in the observed quantum dynamics well before the limit of classical motion is obtained.

We also computed the Lyapunov exponent for the quantum system when its action is sufficiently small that smooth classical dynamics cannot emerge, even for strong measurement [38]. Taking a value of $\hbar=16$, we find that for $k=5 \times 10^{-3}, \lambda=0.029 \pm 0.008$, for $k=0.01, \lambda=0.046 \pm 0.01$ and for $k=0.02, \lambda=0.077 \pm 0.01$. Thus the system is once again chaotic, and 

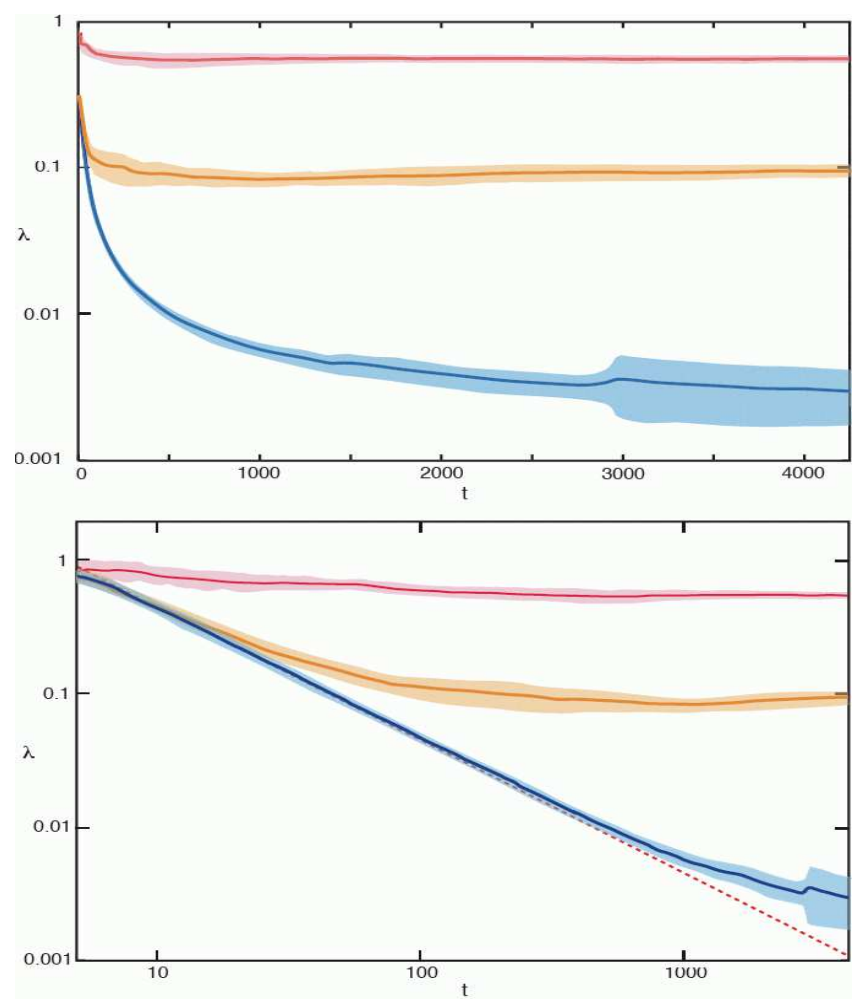

Figure 6. Finite-time Lyapunov exponents $\lambda(t)$ for measurement strengths $k=5 \times$ $10^{-4}, 0.01,10$, averaged over 32 trajectories for each value of $k$ (linear scale in time, top, and logarithmic scale, bottom; bands indicate the standard deviation over the 32 trajectories) [38]. The (analytic) $1 / t$ fall-off at small $k$ values, prior to the asymptotic regime, is evident in the bottom panel. The unit of time is the driving period.

becomes more strongly chaotic the more strongly it is observed. From these striking results, it is clear that there exists a purely quantum regime in which an observed system, while behaving in a fashion quite distinct from its classical limit, nevertheless evolves chaotically with a finite Lyapunov exponent, also distinct from the classical value.

It is worth pointing out that an analogous analysis can also be carried out for a continuously observed classical system. As mentioned previously, an unobserved probabilistic classical system also has provably zero Lyapunov exponent: the average of $x$ for an ensemble of classical particles does not exhibit chaos, due to the linearity of the Liouville equation [40]. If we consider a noiseless observed chaotic classical system - possible since classical measurements are by definition passive (no backaction noise) - then even the weakest meaningful measurement will, over time, localize the probability density, gen- 


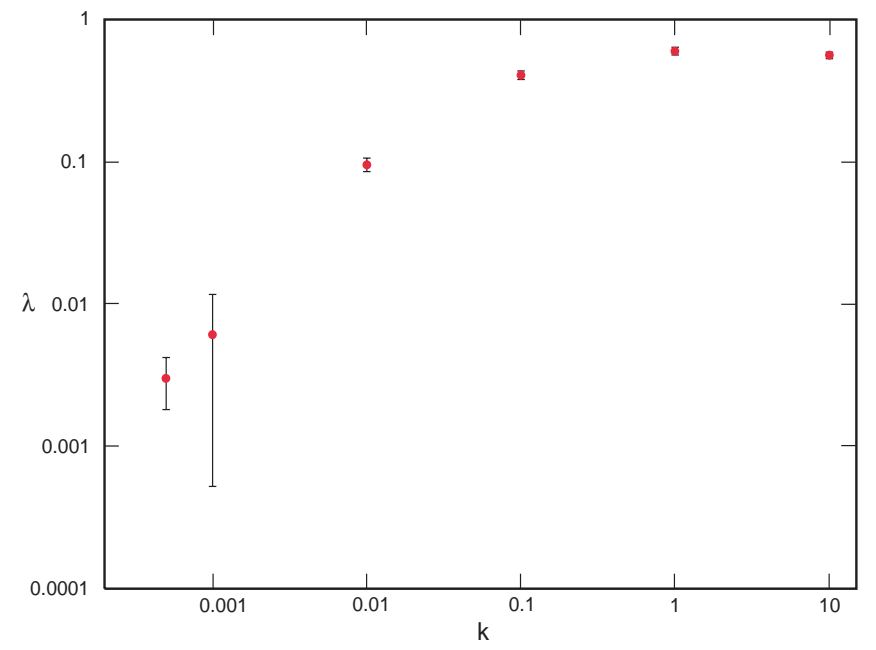

Figure 7. The emergence of chaos [38]: The Lyapunov exponent $\lambda$ as a function of measurement strength $k$. Error-bars follow those of Fig. 6, taken at the final time.

erating an effective trajectory limit, and thus the classical Lyapunov exponent, $\lambda_{C l}$ [40]. Noise can always be be injected into classical systems as an external drive, nevertheless, in the limit of weak noise, the system will once again possess the noiseless exponent $\lambda_{C l}$ : In a classical system the external noise is not connected to the strength of the measurement, so one can simultaneously have strong measurement and weak noise, which, as we have seen, is possible in the quantum theory only under specific conditions [21].

As one way to understand the classical case, we can employ the quantum result as an intermediate step. Consider the quantum Lyapunov exponent at a fixed value of $k$ (where $\lambda<\lambda_{C l}$ ) as in Fig. 7. If the value of $\hbar$ is now reduced, the dynamics of the system must tend to the classical limit as the quantumclassical correspondence inequalities of Ref. [21] are better satisfied. Thus the Lyapunov exponent in the classical limit of quantum theory - which, to a very good approximation, is just classical dynamics driven by weak noise - must tend to $\lambda_{C l}$. If, however, the noise is not weak, an observed classical system, like a quantum system outside the classical regime, will also not be localized, and may well have an exponent different from $\lambda_{C l}$. In addition, one may expect the non-localized quantum and classical evolutions to have quite different Lyapunov exponents, especially when $\hbar$ is large on the scale of the phase space, as quantum and classical evolutions generated by a given nonlinear Hamiltonian are essentially different [12]. The nature of the Lyapunov exponent for nonlocalized classical systems, and its relationship to the exponent for quantum systems is a very interesting open question. 


\section{Concluding Remarks}

To summarize, we have presented a simple analysis of continuously observed classical and quantum dynamical systems. This analysis is in fact required to deal with next-generation experiments and underlies the nascent field of real-time quantum feedback control. Major results include an intuitive and quantitative understanding of the quantum-classical transition. It is pleasing that both the strong and weak forms of the QCT can eventually be understood as a macroscopic limit of observed-system quantum mechanics, i.e., whenever the observed system action $S \gg \hbar$.

Perhaps, most interestingly, we have obtained clear predictions for dynamical chaos in observed quantum systems that are far from the classical regime. We emphasize that the chaos identified here is not merely a formal result even deep in the quantum regime, the Lyapunov exponent can be obtained from measurements on a real system as in near-future cavity QED and nanomechanics experiments [7]. Experimentally, one would use the known measurement record to integrate the SME (5); this provides the time evolution of the mean value of the position. From this fiducial trajectory, given the knowledge of the system Hamiltonian, the Lyapunov exponent can be obtained by following the procedure described here.

\section{Acknowledgments}

SH thanks the organizers of the 16th Florida Workshop in Nonlinear Astronomy and Physics, dedicated to the memory of Henry Kandrup, for their kind invitation to lecture at the meeting. Large-scale parallel computing support from Los Alamos National Laboratory's Institutional Computing Initiative is gratefully acknowledged. This research is supported by the Department of Energy, under contract W-7405-ENG-36.

\section{References}

[1] R. Kosloff and S.A. Rice, J. Chem. Phys. 74, 1340 (1981); J. Manz, J. Chem. Phys. 91, 2190 (1989).

[2] P. Bocchieri and A. Loinger, Phys. Rev. 107, 337 (1957); T. Hogg and B.A. Huberman, Phys. Rev. Lett. 48, 711 (1982).

[3] J.S. Bell, Phys. World, 8, 33 (1990).

[4] B.O. Koopman, Proc. Natl. Acad. Sci. USA 17, 31 (1931).

[5] A. Peres, Quantum Theory: Concepts and Methods (Kluwer, Boston, 1993).

[6] P.S. Maybeck, Stochastic Models, Estimation and Control (Academic Press, New York, 1982); O.L.R. Jacobs, Introduction to Control Theory (Oxford University Press, Oxford, 1993).

[7] H. Mabuchi and A.C. Doherty, Science 298, 1372 (2002); M.D. LaHaye, O. Buu, B. Camarota, and K.C. Schwab, Science 304, 74 (2004). 
[8] See, e.g., J.S. Bell, Speakable and unspeakable in quantum mechanics (Cambridge University Press, New York, 1988).

[9] L.D. Landau and E.M. Lifshitz, Quantum Mechanics: Non-Relativistic Theory (Pergamon Press, New York, 1965).

[10] L.D. Landau and E.M. Lifshitz, Statistical Physics (Pergamon Press, New York, 1980).

[11] K. Hepp, Helv. Phys. Acta 45, 237 (1972); W.H. Zurek, Phys. Rev. D 24, 1516 (1981); ibid 26, 1862 (1982); E. Joos and H.D. Zeh, Z. Phys. B 59, 223 (1985).

[12] S. Habib, K. Jacobs, H. Mabuchi, R. Ryne, K. Shizume and B. Sundaram, Phys. Rev. Lett. 88, 040402 (2002).

[13] E.P. Wigner, Phys. Rev. 40, 749 (1932); V.I. Tatarskii, Usp. Fiz. Nauk 139, 587 (1983) [Sov. Phys. Uspekhi 26, 311 (1983)]; M. Hillery, R.F. O'Connell, M.O. Scully, and E.P. Wigner, Phys. Rep. 106, 121 (1984).

[14] S. Habib, Phys. Rev. D 42, 2566 (1990).

[15] L.P. Kadanoff and G. Baym, Quantum Statistical Mechanics (Addison-Wesley, Redwood City, 1989); R. Zwanzig, Nonequilibrium Statistical Mechanics (Oxford University Press, New York, 2001); K. Blum, Density Matrix Theory and Applications (Plenum Press, New York, 1996).

[16] L. Diosi, Phys. Lett. 129A, 419 (1988); V.P. Belavkin and P. Staszewski, Phys. Lett. 140A, 359 (1989); Y. Salama and N. Gisin, Phys. Lett. 181A, 269 (1993);C.M. Caves and G.J. Milburn, Phys. Rev. A 36, 5543 (1987); H.M. Wiseman and G.J. Milburn, Phys. Rev. A 47, 642(1993); H.J. Carmichael, An Open Systems Approach to Quantum Optics (Springer-Verlag, Berlin, 1993); G.J. Milburn, Quantum Semiclass. Opt. 8, 269 (1996); T.A. Brun, Am. J. Phys. 70, 719 (2002); P. Warszawski and H.M. Wiseman, J. Opt. B 5, 1 (2003).

[17] A.C. Doherty, K. Jacobs, and G. Jungman, Phys. Rev. A 63, 062306 (2001).

[18] D.T. Gillespie, Am. J. Phys. 64, 225 (1996).

[19] See, e.g., D. Mozyrsky and I. Martin, Phys. Rev. Lett. 89, 018301 (2002).

[20] T.P. McGarty, Stochastic Systems and State Estimation (Wiley-Interscience, New York, 1974).

[21] T. Bhattacharya, S. Habib and K. Jacobs, Phys. Rev. Lett. 85, 4852 (2000); Phys. Rev. A 67, 042103 (2003). See also, S. Ghose, P. Alsing, I. Deutsch, T. Bhattacharya, and S. Habib, Phys. Rev. A 69, 052116 (2004).

[22] See, e.g., S. Habib, quant-ph/0406011

[23] B.V. Chirikov, Chaos 1, 95 (1991).

[24] W.A. Lin and L.E. Ballentine, Phys. Rev. Lett. 65, 2927 (1990).

[25] S. Habib, K. Shizume, and W.H. Zurek, Phys. Rev. Lett. 80, 4361 (1998).

[26] B.D. Greenbaum, S. Habib, K. Shizume, and B. Sundaram, Chaos (in press); quant$\mathrm{ph} / 0401174$

[27] G. Lindblad, Comm. Math. Phys. 48, 199 (1976); V. Gorini, A. Kossakowski, and E.C.G. Sudarshan, J. Math. Phys. 17, 821 (1976).

[28] A.O. Caldeira and A.J. Leggett, Phys. Rev. A 31, 1059 (1985).

[29] M.V. Berry, Phil. Trans. Roy. Soc. A 287, 237 (1977); E.J. Heller, J. Chem. Phys. 67, 3339 (1977).

[30] A.R. Kolovsky, Phys. Rev. Lett. 76, 340 (1996). 
[31] V.P. Maslov and M.V. Fedoriuk, Semi-Classical Approximation in Quantum Mechanics (Reidel, Holland, 1981).

[32] M.V. Berry and N.L. Balazs, J. Phys. A 12, 625 (1979).

[33] E.J. Heller and S. Tomsovic, Phys. Today 7, 38 (1993); Phys. Rev. E 47, 282 (1993).

[34] J. Guckenheimer and P. Holmes, Nonlinear Oscillations, Dynamical Systems, and Bifurcations of Vector Fields (Springer-Verlag, Berlin, 1986).

[35] V.P. Belavkin, Comm. Math. Phys. 146, 611 (1992); V.P. Belavkin, Rep. Math. Phys. 43, 405 (1999); A.C. Doherty and K. Jacobs, Phys. Rev. A 60, 2700 (1999); A.C. Doherty, S. Habib, K. Jacobs, H. Mabuchi, and S.-M. Tan, Phys. Rev. A 62, 012105 (2000).

[36] H.M. Wiseman and G.J. Milburn, Phys. Rev. Lett. 70, 548 (1993).

[37] See, e.g., H.M. Wiseman, S. Mancini, and J. Wang, Phys. Rev. A 66, 013807 (2002); R. Ruskov and A.N. Korotkov, Phys. Rev. B 66, 041401(R) (2002); A. Hopkins, K. Jacobs, S. Habib, and K. Schwab, Phys. Rev. B 68, 235328 (2003); D.A. Steck, K. Jacobs, H. Mabuchi, T. Bhattacharya, and S. Habib, Phys. Rev. Lett. 92, 223004 (2004).

[38] S. Habib, K. Jacobs, and K. Shizume, quant-ph/0412159

[39] J.-P. Eckmann and D. Ruelle, Rev. Mod. Phys. 57, 617 (1985).

[40] S. Habib, K. Jacobs, and K. Shizume, in preparation.

[41] A. Wolf, J.B. Swift, H.L. Swinney and J.A. Vastano, Physica 16D, 285 (1985).

[42] S. Habib and R.D. Ryne, Phys. Rev. Lett. 74, 70 (1995). 